\title{
Ultra-soft Sources as Type Ia Supernovae Progenitors
}

\author{
Kelly Lepo and Marten van Kerkwijk
}

Department of Astronomy and Astrophysics, University of Toronto,

50 St. George Street, Toronto, ON M5S 3H4, Canada

email: lepo@astro.utoronto.ca, mhvk@astro.utoronto.ca

\begin{abstract}
Missing from the usual considerations of nuclear burning white dwarfs as Type Ia supernovae progenitors are systems with very higher mass transfer rates, where more material than is needed for steady burning accretes on the white dwarf. This will expand the photosphere of the white dwarf, causing it to emit at longer wavelengths. Thus, we propose the name ultrasoft source (USS) for these objects.

We present a VLT/FLAMES survey looking for USSs in the SMC, selected to be bright in the far UV and with blue far UV-V colors. While we find some unusual objects, and recover known planetary nebulae and WR stars, we detect no objects with strong He II lines, which should be a signature of USSs. This null result either puts an upper limit on the number of USSs in the SMC, or shows that we do not understand what the optical spectra of such objects will look like.

We also discuss the unusual LMC [WN] planetary nebula LMC N66 as a possible example of a USS. It has a luminosity consistent with that expected, and its spectra show incompletely CNO-processed material - strong helium lines, some hydrogen, enhanced nitrogen and depleted carbon. It also shows periodic outbursts. USSs may resemble N66 in quiescence. However, it lacks a FUV excess, contrary to our predictions.
\end{abstract}

Keywords. novae, cataclysmic variables - ultraviolet: stars

\section{Introduction}

As outlined in Nomoto et al. (2007), nuclear burning white dwarfs can be divided into three classes, depending on the accretion rate from the secondary onto the white dwarf $(\dot{M})$. Novae, white dwarfs (WDs) accreting below the steady nuclear burning rate ( $\lesssim 1 \times 10^{-7} M_{\odot} \mathrm{yr}^{-1}$, depending on the mass of the white dwarf), will undergo periodic, explosive nuclear burning.

Within a narrow band of accretion rates $\left(\sim 1-4 \times 10^{-7} M_{\odot} \mathrm{yr}^{-1}\right.$, depending on the mass of the WD), stable nuclear burning occurs on the surface. Unlike in a nova, when fusion is ignited in its base, the thicker hydrogen shell can expand and lift degeneracy enough to allow stable burning. The effective temperature of the white dwarf places the peak of its black body spectrum in the soft X-rays, thus they are referred to as supersoft sources (SSS, van den Heuvel, et al. 1992). Supersoft sources are often considered one of the most promising single degenerate (SD) progenitors of Type Ia supernovae (SNe Ia).

Less considered in discussions of nuclear burning white dwarfs are those that are accreting above the steady nuclear burning rate, but below the Eddington limit $(\sim 4 \times$ $\left.10^{-7}-1 \times 10^{-5} M_{\odot} \mathrm{yr}^{-1}\right)$. These white dwarfs will have a hydrogen burning layer over the degenerate core, with an extended envelope. They will not be supersoft X-ray emitters, since the envelope is thick enough to absorb soft X-rays. The larger photosphere of such objects will move the peak emission into longer wavelengths, likely the (far) UV. For lack of a better name, we dub these ultra-soft sources (USSs). 
There are two general ideas for what a USS might look like. The first is that the WD will have an extended envelope. Such an object may also lose mass from the L2 Lagrange point and/or form a common envelope (Nomoto et al. 1979, Iben 1988). Most cataclysmic variables have periods of about 80 min to 10 hours, limiting the WD's envelope to its $\sim 1 R_{\odot}$ Roche lobe. If the system has not formed a common envelope, the WD component will appear as a very small OB giant.

The second possibility is that the WD will lose mass through optically thick winds (Hachisu et al. 1996). Depending on the mass loss rate and the accretion rate, the WD may or may not fill its Roche lobe. These may appear like Wolf-Rayet stars or [WR] planetary nebulae. Hachisu \& Kato (2003a) and Hachisu \& Kato (2003b) use a similar model to explain V Sge and V Sge-like stars.

\section{A Hunt for USSs in the SMC}

The Magellanic Clouds are relatively close to us and have dense stellar fields. There is also a low column density between the Magellanic clouds and us, which will not absorb UV photons as happens in the Milky Way. They thus are ideal places to look for USSs.

Because the Magellanic Clouds are too bright for GALEX, we turn to a relatively obscure telescope, the Ultraviolet Imaging Telescope (UIT), which flew on two space shuttle missions in late 1990 and early 1995. UIT imaged both Magellanic Clouds in several FUV filters, including B5 at $162 \mathrm{~nm}$ (Cornett et al. 1997). We redid the astrometry and crossidentified sources with the Magellanic Cloud Photometric Survey (MCPS, Zaritsky et al. 2002) to get more accurate positions for sources in the UIT catalog. Since the UIT observations cover the entire the central core of the SMC, while the LMC observations are in scattered fields, we chose to use the SMC data to simplify followup observations.

Using Equation 4 from Di Stefano (2010), we can estimate the number of accreting WDs expected in the SMC, based on the SNe Ia rate of the galaxy (as determined by its blue luminosity). Using similar values to Di Stefano, and $L_{B}=4.4 \times 10^{8}$ for the SMC, we find that $N_{a c c}$ (the number of accreting white dwarfs needed to reproduce the Type Ia rate) is 33-132 for the SMC. There are four known SSS in the SMC (Greiner 2000). Assuming a single degenerate progenitor, there should be dozens of missing progenitors in the SMC. While USSs will probably not make up all of the missing progenitors (other good SD candidates include recurrent novae and symbiotic stars), WDs with high accretion rates should exist.

Figure 1 is a color magnitude diagram of $m_{162}-\mathrm{v}$ vs v, using values from the FUV and optical catalogs. The blue edge of the central clump of stars corresponds to the position of the main sequence calculated by Cornett et al. (1997). Stars to the red side of the central clump are either evolved or reddened. Stars on the blue side of the central clump have an unexplained UV excess. This is the region where we expect to find USS.

We took optical spectra of a majority of the objects outside of the central clump of Figure 1, using the multi-object spectrometer VLT/FLAMES. We also took spectra of objects within the central clump using any extra fibers. We had five fields, which overlapped slightly, with $\sim 110$ objects in each field. Two fields were observed twice because the first observations were not taken within specified seeing conditions, but even the non-ideal observations turned out to be usable. In the end, we had 496 unique objects and 745 spectra.

As expected, the majority of objects in the central main sequence clump in Figure 1, were normal OB stars along with Be and B [e] stars. Stars to the red side of central clump (that had abnormally low FUV emission) were generally O, B and A giants. 


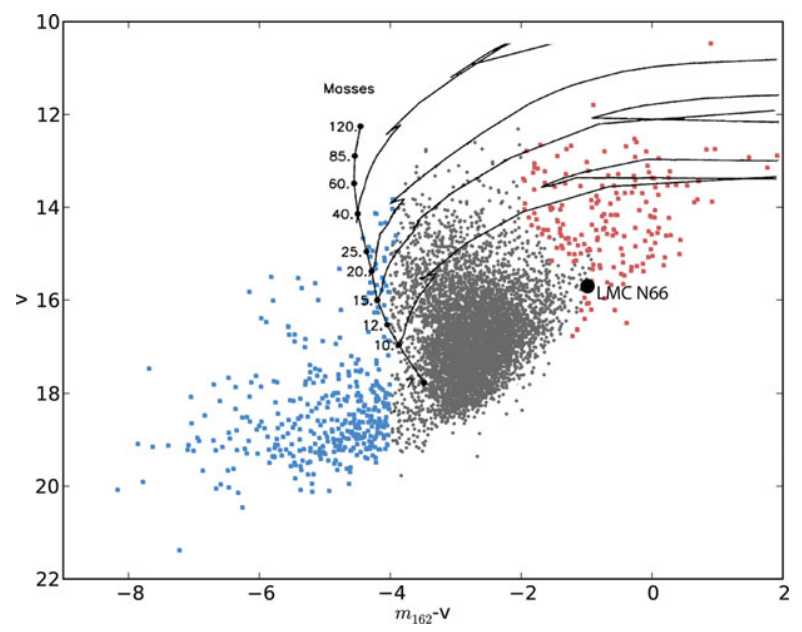

Figure 1. Color magnitude diagram of $\mathrm{m}_{162}-\mathrm{v}$ vs $\mathrm{v}$, using values from UIT and MCPS. There are 5723 points in the central clump (circles), 182 points redder than the central clump (squares) and 396 points bluer than the central clump (squares). Overlaid is Figure 4 from Cornett et al. (1997), showing evolutionary tracks of OB stars. Also marked is the location of LMC N66, using values from the 25 April 1995 UIT observation and an April 1995 optical observation from Peña et al. (1996). At least during outburst, LMC N66 does not have a UV excess.

Stars to the blue side of the central clump (with a FUV excess) were generally: (a) mismatches between the FUV and optical catalogs, (b) stars that seemed to have bad UVB measurements in the optical catalog, (c) objects that look like faint B stars, and (d) main sequence stars with nebular lines. Most of the main sequence stars were found near the edge of the central clump, so they likely belonged to the "main sequence" group.

We did recover some known objects that are similar to what we expect a USS to look like: SMC-SMP 8 a [WC 8] planetary nebula with an unusual core, 2MASS J005207387235385 a (not particularly well studied) WR star, and several objects that look like planetary nebulae. However, there were no objects with strong He II 4686 emission lines, which are found in the spectra of SSSs, V Sge-like stars, and the LMC [WN] planetary nebula N66. It is likely that the hot WD in a USS would produce ionized helium emission lines, meaning it is unlikely we observed any USSs. This null result either puts an upper limit on the number of USS in the SMC, or shows that we do not understand what the optical spectra of such objects will look like.

\section{LMC N66: A Possible USS?}

LMC N66 (also known as WS 35 and SMP 83) is a unusual early [WN] planetary nebula located in the LMC. It undergoes periodic outbursts, where its luminosity increases by about an order of magnitude for a period of years. Its pre-outburst luminosity $\left(\log \left[L / L_{\odot}\right]=4.5\right)$ is higher than known [WC] planetary nebulae, and its outburst luminosity $\left(\log \left[L / L_{\odot}\right]=5.4\right)$ is on par with galactic WN stars (Hamann et al. 2003).

Two outbursts have been observed, with peak luminosities occurring in 1994 and 2007 (Peña et al. 1995, Hamann et al. 2003, Peña et al. 2008). There is no evidence in archival data that any outbursts occurred from 1955 to 1990 (Hamann et al. 2003).

Spectra of LMC N66 show incompletely CNO-processed material — strong helium emission lines, some hydrogen, enhanced nitrogen and depleted carbon. From the width of the lines one infers a low terminal wind speed. The model that has the fewest contradictions with the observed properties LMC N66, is that the central object of the 
planetary nebula is a low-mass binary, with a WD primary and a non-degenerate secondary. The WD rapidly accretes mass, and loses some to winds. The outbursts - hard to explain with a single star model - may be due to helium shell flashes on the white dwarf (Hamann et al. 2003).

Assuming the system is a WD and a normal star, models by Hamann et al. (2003) put the radius of the photosphere of the WD at $0.52-1.38 R_{\odot}$. This is about what one would expect for the Roche lobe of a WD in a close binary. They find the mass loss rate of the object varies from $\dot{M}=10^{-5.7}$ during quiescence to $\dot{M}=10^{-5.0} M_{\odot} \mathrm{yr}^{-1}$ during outburst. This would put the WD in the high $\dot{M}$ regime. Both the radius and $\dot{M}$ calculations are consistent with our ideas of USSs.

LMC N66 was observed by UIT on 25 April 1995, but it was so close to the edge of the photographic plate that is was not included in the catalog by Parker et al. (1998). We redid the photometry of the entire UIT LMC field that LMC N66 was in, scaled our uncalibrated magnitudes so that they matched the published, calibrated ones, and then calculated the magnitude of LMC N66. We find $\mathrm{m}_{162}=15.22$. When UIT observed LMC N66, the nebula had passed the peak of its outburst, but was not yet in quiescence. Peña et al. (1996) also observed LMC N66 in April 1995 at optical wavelengths and find $m_{v}=16.2 \pm 0.2$.

Putting those two observations together and plotting on Figure 1, we see that LMC N66 falls to the red side of the central clump, meaning that instead of having a UV excess, as was expected, the object has less FUV emission than main sequence stars. However, since we only have one observation during the end of an outburst, this may not be applicable during quiescence or other parts of the outburst cycle. In addition, since LMC N66 was near the edge of the plate, its flux may have been underestimated.

Although Hamann et al. (2003) suggest that the best model of LMC N66 is for a WD binary with a low mass companion, very little work has been done to verify that the core of LMC N66 is in fact a binary. High resolution spectroscopy to look for radial velocity variations and photometry to look for $\sim 1$ day variations in the light curve might help to confirm the binary model.

\section{References}

Cornett, R. H., Greason, M. R., Hill, J. K., Parker, J. W., \& Waller, W. H. 1997 AJ, 113, 1011

Di Stefano, R. 2010, ApJ, 712, 782

Greiner, J. 2000, New Astron., 5, 137

Hachisu, I. \& Kato, M. 2003a, ApJ, 590, 445

Hachisu, I. \& Kato, M. 2003b, ApJ, 598, 527

Hachisu, I., Kato, M., \& Nomoto, K. 1996, ApJ, 470, L97

Hamann, W.-R., Peña, M., Gräfener, G., \& Ruiz, M. T. 2003, A\&\&A, 409, 969

Iben, I. Jr. 1988 ApJ, 324, 355

Nomoto, K., Nariai, K., \& Sugimoto, D. 1979, PASJ, 31, 287

Nomoto, K., Saio, H., Kato, M., \& Hachisu, I. 2007, ApJ, 663, 1269

Nomoto, K., Saio, H., Kato, M., \& Hachisu, I. 2007, ApJ, 663, 1269

Parker, J. W., Hill, J. K., Cornett, R. H., Hollis, J., Zamkoff, E., Bohlin, R. C., O'Connell, R. W., Neff, S. G., Roberts, M. S., Smith, A. M., \& Stecher, T. P. 1998, AJ, 116, 180

Peña, M., Peimbert, M., Torres-Peimbert, S., Ruiz, M. T., \& Maza, J. 1995, ApJ, 441, 343

Peña, M., Peimbert, M., Torres-Peimbert, S., Ruiz, M. T., \& Maza, J. 1996, Ap $\mathscr{E} S S, 238,55$

Peña, M., Ruiz, M. T., Maza, J., Rojo P., Torres-Peimbert, S., \& Hamann, W.-R. 2008, ApJ, $680 \mathrm{~L}, 109$

van den Heuvel, E. P. J., Bhattacharya, D., Nomoto, K., \& Rappaport, S. A. 1992, A\&\&A, 262, 97

Zaritsky D., Harris, J., Thompson, I. B., Grebel, E. K., \& Massey, P. 2002 AJ, 123, 855 